\title{
Almost sure exponential stability for time-changed stochastic differential equations
}

\author{
Yongxiang Zhu ${ }^{\mathrm{a}}$, Min Zhu $\mathrm{h}^{\mathrm{a}, \mathrm{b}, *}$, Junping $\mathrm{Li}^{\mathrm{b}}$ \\ ${ }^{a}$ College of Traffic Engineering, Hunan University of Technology, Zhuzhou, Hunan, 412007, China. \\ ${ }^{b}$ School of Mathematics and Statistics, Central South University, Changsha, Hunan, 410083, China.
}

Communicated by X. Z. Liu

\begin{abstract}
Some sufficient conditions for almost sure exponential stability of solutions to time-changed stochastic differential equations (SDEs) are presented. The principle technique of our investigation is to construct a proper Lyapunov function and carry out generalized Lyapunov methods to time-changed SDEs. In contrast to the almost sure exponential stability in existing articles, we present new results on the stability of solutions to time-changed SDEs. Finally, an example is given to demonstrate the effectiveness of our work. (c)2017 All rights reserved.
\end{abstract}

Keywords: Time-changed stochastic differential equations, almost sure exponential stability, time-changed Brownian motion. 2010 MSC: $60 \mathrm{H} 20,60 \mathrm{H} 30$.

\section{Introduction}

Time change and its related stochastic calculus have been widely used to study dynamics of various complex stochastic processes arising in anomalous diffusion. We refer to [4-6, 10, 13, 14] and the references therein. Meanwhile, many excellent works have been done on time-changed stochastic differential equations (SDEs), see [7, 9, 11]. Especially, Kobayashi [11] extended classical SDEs to a lager class SDEs driven by time-changed semimartingales and derived the corresponding time-changed Itô formula, which provided the new perspective for the study of SDEs. As it is well-known to all that the study of stability properties of SDEs have been one of the most active areas in stochastic analysis, involved in several fields such as physics, mechanical engineering, biology, and economics (see $[1-3,8,15,16,19,22,23]$ and the references therein). Lyapunov's method is in general available to obtain sufficient conditions for the stability of solutions. Very recently, within the new framework of what Kobayashi [11] had set, the stability theory of time-changed SDEs has started attracting much attention of many researchers. By means of the generalized Lyapunov method, $\mathrm{Wu}$ [21] established sufficient conditions for stability of solutions of time-changed SDEs in different senses, such as stochastic stability, stochastically asymptotic stability, and

\footnotetext{
*Corresponding author $\mathrm{Li})$

Email addresses: zyx1998@sina.com (Yongxiang Zhu), zhumin0107@csu.edu.cn (Min Zhu), jpli@mail.csu.edu.cn (Junping doi:10.22436/jnsa.010.11.35
} 
globally stochastically asymptotic stability. Likewise, Wu [20] investigated stable behaviors of solutions for different time-changed systems, such as exponential sample-path stability, $p$-th moment asymptotic stability, and p-th moment exponential stability. Nane and Ni [17] studied stabilities of SDEs driven by time-changed Lévy noise in both probability and moment sense. So far there has no work presented on the almost sure exponential stability of solutions to time-changed SDEs. Motivated by the above discussion, in this work we shall study the almost sure exponential stability problem for a class of time-changed SDEs.

The rest of this paper is structured as follows. In Section 2 we recall some preliminary results. Section 3 shows some criteria for almost sure exponential stability and in the last section, to show the effectiveness of our theories, an illustrative example is provided.

\section{Preliminary}

Let $(\Omega, \mathcal{F}, \mathcal{P})$ be a complete probability space with a filtration $\left\{\mathcal{F}_{t}\right\}_{t \geqslant 0}$ satisfying the usual conditions. $\{\mathrm{B}(\mathrm{t})\}_{\mathrm{t} \geqslant 0}$ is a real-valued Brownian motion defined on the stochastic basis $\left(\Omega, \mathcal{F},\left\{\mathcal{F}_{t}\right\}_{t \geqslant 0}, \mathcal{P}\right)$. Define the inverse subordinator by

$$
E(t)=\inf \{\tau>0: U(\tau)>t\}, \quad t \geqslant 0,
$$

where $\{\mathrm{U}(\tau)\}_{\tau \geqslant 0}$ is an $\mathcal{F}_{\mathrm{t}}$-adapted subordinator with Laplace transform $\mathbb{E}\left(\mathrm{e}^{-\mathfrak{u} u(\tau)}\right)=\mathrm{e}^{-\tau \psi(u)}$, where $\psi(u)=b u+\int_{0}^{\infty}\left(1-e^{-u y}\right) v(d y)(u>0)$ with $b \geqslant 0$ and $\int_{0}^{\infty}(x \wedge 1) v(d y)<\infty$. Assume that the Lévy measure $v$ is infinite. It implies that the subordinator $U$ has strictly increasing paths, and then $E$ has continuous, nondecreasing paths. We assume that the Brownian motion $\{B(t)\}_{t \geqslant 0}$ is independent of $\{\mathrm{E}(\mathrm{t})\}_{\mathrm{t} \geqslant 0}$. Set

$$
\mathcal{F}_{t}=\bigcap_{u \rightarrow t} \sigma(\{B(y): 0 \leqslant y \leqslant u\},\{E(t): t \geqslant 0\}) .
$$

Then $\left\{\mathcal{F}_{t}\right\}_{t} \geqslant 0$ is right continuous, $\{\mathrm{B}(\mathrm{t})\}_{\mathrm{t} \geqslant 0}$ is a martingale with respect to the filtration $\left\{\mathcal{F}_{t}\right\}_{t \geqslant 0}$. Thus $\left\{\mathcal{G}_{t}\right\}_{t \geqslant 0}$ is a well-defined filtration where $\mathcal{G}_{t}=\mathcal{F}_{E(t)}$, and also satisfies the usual condition since the rightcontinuity of $\left\{\mathcal{F}_{t}\right\}_{t} \geqslant 0$. According to Theorem 1 in [14], the time-changed Brownian motion $B(E(t))$ is a square integrable martingale with respect to the filtration $\left\{\mathcal{G}_{t}\right\}_{t \geqslant 0}$. Let $\Phi: \mathbb{R}_{+} \rightarrow \mathbb{R}$ be a predictable, $\mathcal{G}_{\mathrm{t}}$-adapted process such that

$$
\mathbb{E} \int_{0}^{t}|\Phi(s)|^{2} \mathrm{dE}(s)<\infty, \quad t>0 .
$$

Then we can define the real-valued stochastic integral

$$
\int_{0}^{t} \Phi(s) d B(E(s))
$$

which is a continuous square-integrable martingale. For details of the construction of stochastic integrals, consult [18].

In this work, we investigate the following SDE driven by time-changed Brownian motion

$$
d X(t)=f(t, E(t), X(t)) d t+g(t, E(t), X(t)) d E(t)+\sigma(t, E(t), X(t)) d B(E(t))
$$

with initial value $X(0)=x_{0}$, and $f, g, \sigma$ are real-valued functions, defined on $\mathbb{R}_{+} \times \mathbb{R}_{+} \times \mathbb{R}$.

Throughout this paper, for the existence and uniqueness of the solution to (2.1), we shall impose the following assumptions.

Assumption 2.1 (Lipschitz condition). There exists a positive constant K such that

$$
\left|f\left(t_{1}, t_{2}, x\right)-f\left(t_{1}, t_{2}, y\right)\right|+\left|g\left(t_{1}, t_{2}, x\right)-g\left(t_{1}, t_{2}, y\right)\right|+\left|\sigma\left(t_{1}, t_{2}, x\right)-\sigma\left(t_{1}, t_{2}, y\right)\right| \leqslant K|x-y|
$$

for all $t_{1}, t_{2} \in \mathbb{R}_{+}$and $x, y \in \mathbb{R}$. 
Assumption 2.2. If $X(t)$ is a càdlàg and $\mathcal{G}_{t}$-adapted process, then

$$
f(t, E(t), X(t)), g(t, E(t), X(t)), \sigma(t, E(t), X(t)) \in \mathcal{L}\left(\mathcal{G}_{t}\right),
$$

where $\mathcal{L}\left(\mathcal{G}_{t}\right)$ denotes the class of càglàd and $\mathcal{G}_{t}$-adapted processes.

According to Lemma 4.1 in [11], (2.1) admits a unique solution $X(\cdot)$ which is a $\mathcal{G}_{\mathrm{t}}$-adapted process.

Definition 2.3. The solution of time-changed SDE (2.1) is said to be almost surely exponentially stable if there exists a $\lambda>0$ such that

$$
\limsup _{t \rightarrow \infty} \frac{1}{t} \log |X(t)| \leqslant-\lambda \quad \text { a.s.. }
$$

We now introduce some notations. Let two operators $L_{1}$ and $L_{2}$ acting on $C^{1,1,2}\left(\mathbb{R}_{+} \times \mathbb{R}_{+} \times \mathbb{R} ; \mathbb{R}_{+}\right)$valued functions as follows:

$$
L_{1} V\left(t_{1}, t_{2}, x\right)=V_{t_{1}}\left(t_{1}, t_{2}, x\right)+V_{x}\left(t_{1}, t_{2}, x\right) f\left(t_{1}, t_{2}, x\right)
$$

and

$$
L_{2} V\left(t_{1}, t_{2}, x\right)=V_{t_{2}}\left(t_{1}, t_{2}, x\right)+V_{x}\left(t_{1}, t_{2}, x\right) g\left(t_{1}, t_{2}, x\right)+\frac{1}{2} V_{x x} \sigma^{2}\left(t_{1}, t_{2}, x\right),
$$

where

$$
\begin{aligned}
V_{t_{1}}\left(t_{1}, t_{2}, x\right) & =\frac{\partial V\left(t_{1}, t_{2}, x\right)}{\partial t_{1}}, & V_{t_{2}}\left(t_{1}, t_{2}, x\right) & =\frac{\partial V\left(t_{1}, t_{2}, x\right)}{\partial t_{2}} \\
V_{x}\left(t_{1}, t_{2}, x\right) & =\frac{\partial V\left(t_{1}, t_{2}, x\right)}{\partial x}, & V_{x x}\left(t_{1}, t_{2}, x\right) & =\frac{\partial^{2} V\left(t_{1}, t_{2}, x\right)}{\partial x^{2}} .
\end{aligned}
$$

\section{Almost sure exponential stability}

This section concludes some criteria for almost sure exponential stability of solution to (2.1). To establish the results on the almost sure exponential stability, we impose the following assumption.

Assumption 3.1. For all $t>0$, if $x_{0} \neq 0$ in $\mathbb{R}_{+}, \mathbb{P}\left\{X\left(t, x_{0}\right) \neq 0\right.$, on $\left.t \geqslant 0\right\}=1$.

The following lemma tells that Assumption 3.1 holds under some conditions.

Lemma 3.2. Assume that for any $\theta>0$ there exists a positive constant $\mathrm{K}_{\theta}$ such that

$$
\left|f\left(t_{1}, t_{2}, x\right)\right|+\left|g\left(t_{1}, t_{2}, x\right)\right|+\left|\sigma\left(t_{1}, t_{2}, x\right)\right| \leqslant K_{\theta}|x| .
$$

If $|x| \leqslant \theta$ and $t \geqslant 0$, then for all $x_{0} \neq 0$ in $\mathbb{R}_{+}$,

$$
\mathbb{P}\left\{X\left(t, x_{0}\right) \neq 0, \text { on } t \geqslant 0\right\}=1 \text {. }
$$

Proof. We shall apply the method used in the argument of [16] to prove this result. Assume that (3.1) is false. Then there would exist some $x_{0} \neq 0$ such that $\mathbb{P}(\tau<\infty)>0$ where $\tau:=\inf \{t \geqslant 0: X(t)=0\}$ which stands for the first time of zero of the corresponding solution. We can choose a pair of constants $T>0$ and $\bar{\theta}>1$ large enough so that $\mathbb{P}(B)>0$, where

$$
B=\{\omega \in \Omega: \tau(\omega) \leqslant T \text { and }|X(t)| \leqslant \bar{\theta}-1, \text { for all } 0 \leqslant t \leqslant \tau(\omega)\} .
$$

Let $V\left(t_{1}, t_{2}, x\right)=|x|^{-1}$ and $\alpha=K_{\theta}\left(2+K_{\theta}\right)$. If $0<|x| \leqslant \theta$, applying the time-changed Itô formula (see [21]), it follows that

$$
L_{1} V(t, E(t), X(t))=-|X(t)|^{-2} f(t, E(t), X(t)) \leqslant K_{\theta}|X(t)|^{-1} \leqslant \alpha V(t, E(t), X(t))
$$


and

$$
\begin{aligned}
\mathrm{L}_{2} \mathrm{~V}(\mathrm{t}, \mathrm{E}(\mathrm{t}), X(\mathrm{t})) & =-|X(\mathrm{t})|^{-2} g(t, \mathrm{E}(\mathrm{t}), X(\mathrm{t}))+|X(t)|^{-3} \sigma^{2}(\mathrm{t}, \mathrm{E}(\mathrm{t}), X(\mathrm{t})) \\
& \leqslant \mathrm{K}_{\theta}|X(t)|^{-1}+\mathrm{K}_{\theta}^{2}|X(t)|^{-1} \leqslant \alpha \mathrm{V}(\mathrm{t}, \mathrm{E}(\mathrm{t}), X(\mathrm{t})) .
\end{aligned}
$$

Define the following family of stopping times, for any $0<\varepsilon<\left|x_{0}\right|$,

$$
\tau_{\varepsilon}=\inf \{t \geqslant 0:|X(t)| \leqslant \varepsilon \text { or }|X(t)| \geqslant \bar{\theta}\} .
$$

By the time-changed Itô formula applied to $Z\left(t_{1}, t_{2}, x\right)=e^{-\alpha\left(t_{1}+t_{2}\right)} V\left(t_{1}, t_{2}, x\right)$ we obtain

$$
\begin{aligned}
\exp \{ & \left.-\alpha\left(\left(\tau_{\varepsilon} \wedge \mathrm{T}\right)+\mathrm{E}\left(\tau_{\varepsilon} \wedge \mathrm{T}\right)\right)\right\} \mathrm{V}\left(\tau_{\varepsilon} \wedge \mathrm{T}, \mathrm{E}\left(\tau_{\varepsilon} \wedge \mathrm{T}\right), \mathrm{X}\left(\tau_{\varepsilon} \wedge \mathrm{T}\right)\right) \\
= & \mathrm{V}\left(0,0, \mathrm{x}_{0}\right)-\int_{0}^{\tau_{\varepsilon} \wedge \mathrm{T}} \alpha \mathrm{e}^{-\alpha(s+\mathrm{E}(s))} \mathrm{V}(\mathrm{s}, \mathrm{E}(\mathrm{s}), \mathrm{X}(\mathrm{s})) \mathrm{d} s-\int_{0}^{\tau_{\varepsilon} \wedge \mathrm{T}} \alpha \mathrm{e}^{-\alpha(s+\mathrm{E}(\mathrm{s}))} \\
& \times \mathrm{V}(\mathrm{s}, \mathrm{E}(\mathrm{s}), \mathrm{X}(\mathrm{s})) \mathrm{dE}(\mathrm{s})+\int_{0}^{\tau_{\varepsilon} \wedge \mathrm{T}} \mathrm{e}^{-\alpha(\mathrm{s}+\mathrm{E}(\mathrm{s}))} \mathrm{L}_{1} \mathrm{~V}(\mathrm{~s}, \mathrm{E}(\mathrm{s}), \mathrm{X}(\mathrm{s})) \mathrm{d} s \\
& +\int_{0}^{\tau_{\varepsilon} \wedge \mathrm{T}} \mathrm{e}^{-\alpha(s+\mathrm{E}(\mathrm{s}))} \mathrm{L}_{2} \mathrm{~V}(\mathrm{~s}, \mathrm{E}(\mathrm{s}), \mathrm{X}(\mathrm{s})) \mathrm{dE}(\mathrm{s}) \\
& +\int_{0}^{\tau_{\varepsilon} \wedge \mathrm{T}} \mathrm{e}^{-\alpha(s+\mathrm{E}(\mathrm{s}))}\left(-|\mathrm{X}(\mathrm{s})|^{-2}\right) \sigma(s, \mathrm{E}(\mathrm{s}), \mathrm{X}(\mathrm{s})) \mathrm{dB}(\mathrm{E}(\mathrm{s})) .
\end{aligned}
$$

From [13] and [12], we have

$$
\mathbb{E} \int_{0}^{\tau_{\varepsilon} \wedge T} \mathrm{e}^{-\alpha(s+E(s))}\left(-|X(s)|^{-2}\right) \sigma(s, E(s), X(s)) d B(E(s))=0 .
$$

Therefore, taking expectation on both sides of (3.4), together with (3.2) and (3.3), yields that

$$
\mathbb{E}\left[\exp \left\{-\alpha\left(\left(\tau_{\varepsilon} \wedge T\right)+E\left(\tau_{\varepsilon} \wedge T\right)\right)\right\} V\left(\tau_{\varepsilon} \wedge T, E\left(\tau_{\varepsilon} \wedge T\right), X\left(\tau_{\varepsilon} \wedge T\right)\right)\right] \leqslant V\left(0,0, x_{0}\right) .
$$

If $\omega \in B$, then $\tau_{\varepsilon}(\omega) \leqslant T$ and $\left|X\left(\tau_{\mathcal{\varepsilon}}(\omega)\right)\right| \leqslant \varepsilon$. Then

$$
\mathbb{E}\left[\mathrm{e}^{-\alpha(\mathrm{T}+\mathrm{E}(\mathrm{T}))} \mathcal{\varepsilon}^{-1} \mathrm{I}_{\mathrm{B}}\right] \leqslant \mathbb{E}\left[\mathrm{e}^{-\alpha\left(\tau_{\varepsilon}+\mathrm{E}\left(\tau_{\varepsilon}\right)\right)}\left|\mathrm{X}\left(\tau_{\varepsilon}(\omega)\right)\right|^{-1} \mathrm{I}_{\mathrm{B}}\right] \leqslant \mathrm{V}\left(0,0, x_{0}\right) .
$$

In addition, according to the reverse Hölder inequality:

$$
|\mathbb{E}(X Y)| \geqslant\left(\mathbb{E}|X|^{p}\right)^{\frac{1}{p}}\left(\mathbb{E}|Y|^{-\frac{1}{1-p}}\right)^{\frac{p-1}{p}}, \quad 0<p<1,
$$

we have

$$
\varepsilon^{-1} \mathrm{e}^{-\alpha \mathrm{T}}(\mathbb{P}(\mathrm{B}))^{2}\left[\mathbb{E}\left[\mathrm{e}^{\alpha \mathrm{E}(\mathrm{T})}\right]\right]^{-1} \leqslant \mathbb{E}\left[\mathrm{e}^{-\alpha(\mathrm{T}+\mathrm{E}(\mathrm{T}))} \mathcal{\varepsilon}^{-1} \mathrm{I}_{\mathrm{B}}\right] .
$$

Moreover, in terms of Lemma 8 in $[9], \mathbb{E}\left[\mathrm{e}^{\alpha \mathrm{E}(\mathrm{T})}\right]$ is finite for any $\alpha \in \mathbb{R}, T>0$. Hence, for all $\varepsilon>0$,

$$
\mathbb{P}(\mathrm{B}) \leqslant \varepsilon \mathrm{e}^{\alpha \mathrm{T}} \mathbb{E}\left[\mathrm{e}^{\alpha \mathrm{E}(\mathrm{T})}\right]\left|x_{0}\right|^{-1} .
$$

Let $\varepsilon \rightarrow 0$, then it follows that $\mathbb{P}(B)=0$ which contradicts the definition of the set $\mathrm{B}$ and the required result follows.

Remark 3.3. Lemma 3.2 tells us that Assumption 3.1 is satisfied if $f\left(t_{1}, t_{2}, 0\right)=g\left(t_{1}, t_{2}, 0\right)=\sigma\left(t_{1}, t_{2}, 0\right)=0$ and Assumption 2.1 are true. But generally speaking, Assumption 2.1 is required in order to have a unique solution, and $f\left(t_{1}, t_{2}, 0\right)=g\left(t_{1}, t_{2}, 0\right)=\sigma\left(t_{1}, t_{2}, 0\right)=0$ can be assumed to be true in the study of stability of solutions. 
Theorem 3.4. Let Assumption 3.1 hold. If $\mathrm{V}\left(\mathrm{t}_{1}, \mathrm{t}_{2}, x\right) \in \mathrm{C}^{1,1,2}\left(\mathbb{R}_{+} \times \mathbb{R}_{+} \times \mathbb{R} ; \mathbb{R}_{+}\right)$and some constants $\mathrm{p}>0$, $\mathrm{c}_{1}>0$, and $\mathrm{c}_{2} \geqslant 0$ satisfy:

(1) $|x|^{p} \leqslant V\left(t_{1}, t_{2}, x\right)$;

(2) $\mathrm{L}_{1} \mathrm{~V}\left(\mathrm{t}_{1}, \mathrm{t}_{2}, x\right) \leqslant-\mathrm{c}_{1} \mathrm{~V}\left(\mathrm{t}_{1}, \mathrm{t}_{2}, x\right)$;

(3) $L_{2} V\left(t_{1}, t_{2}, x\right) \leqslant-c_{2} V\left(t_{1}, t_{2}, x\right)$;

(4) $\mathrm{E}(\mathrm{t})$ is asymptotically slower than $\mathrm{t}$, i.e., $\lim _{\mathrm{t} \rightarrow \infty} \frac{\mathrm{E}(\mathrm{t})}{\mathrm{t}}=0$ a.s.,

then for all $x_{0} \in \mathbb{R}$ and $t \geqslant 0$

$$
\limsup _{t \rightarrow \infty} \frac{1}{t} \log |X(t)| \leqslant-\frac{c_{1}}{p} \text { a.s.. }
$$

Proof. Fix $x_{0} \neq 0$. By Assumption 3.1, for all $t \geqslant 0, X(t) \neq 0$ almost surely. Thus, for all $t \geqslant 0$ one can apply the time-changed Itô formula to show

$$
\begin{aligned}
\log V(t, E(t), X(t))= & \log V\left(0,0, x_{0}\right)+\int_{0}^{t} \frac{L_{1} V(s, E(s), X(s))}{V(s, E(s), X(s))} d s \\
& +\int_{0}^{t} \frac{L_{2} V(s, E(s), X(s))}{V(s, E(s), X(s))} d E(s)-\frac{1}{2} \int_{0}^{t} \frac{V_{x}^{2}(s, E(s), X(s))}{V^{2}(s, E(s), X(s))} \\
& \times \sigma^{2}(s, E(s), X(s)) d E(s)+\int_{0}^{t} \frac{V_{x}(s, E(s), X(s))}{V(s, E(s), X(s))} \times \sigma(s, E(s), X(s)) d B(E(s)) .
\end{aligned}
$$

Let

$$
M(t)=\int_{0}^{t} \frac{V_{x}(s, E(s), X(s))}{V(s, E(s), X(s))} \sigma(s, E(s), X(s)) d B(E(s)) .
$$

Using lemma 6.2 in [15] with $g(t)=t, \tau_{k}=k, \gamma_{k}=1$, and $\theta=2$, we deduce that, for almost all $\omega \in \Omega$, there exists an integer $k_{0}(\omega)$ such that for all $k>k_{0}$

$$
M(t) \leqslant \frac{1}{2}\langle M(t)\rangle+2 \log t, 0 \leqslant t \leqslant k .
$$

Note

$$
\langle M(t)\rangle=\int_{0}^{t} \frac{V_{\chi}^{2}(s, E(s), X(s))}{V^{2}(s, E(s), X(s))} \sigma^{2}(s, E(s), X(s)) d E(s) .
$$

So, for all $0 \leqslant t \leqslant k, k \geqslant k_{0}$,

$$
M(t) \leqslant \frac{1}{2} \int_{0}^{t} \frac{V_{\chi}^{2}(s, E(s), X(s))}{V^{2}(s, E(s), X(s))} \sigma^{2}(s, E(s), X(s)) d E(s)+2 \log k \text { a.s.. }
$$

Substituting this into (3.5), together with conditions (2) and (3) gives, for any $0 \leqslant t \leqslant k, k \geqslant k_{0}$,

$$
\log V(t, E(t), X(t)) \leqslant \log V\left(0,0, x_{0}\right)-c_{1} t-c_{2} E(t)+2 \log k \text { a.s.. }
$$

This along with condition (1) implies, for $k-1 \leqslant t \leqslant k, k \geqslant k_{0}$,

$$
\begin{aligned}
\frac{1}{t} \log |X(t)| \leqslant \frac{1}{p t} \log V(t, E(t), X(t)) & \leqslant \frac{1}{p t}\left(\log V\left(0,0, x_{0}\right)-c_{1} t-c_{2} E(t)+2 \log k\right) \\
& \leqslant \frac{\log V\left(0,0, x_{0}\right)-c_{1} t-c_{2} E(t)}{p t}+\frac{2 \log k}{p(k-1)} \text { a.s.. }
\end{aligned}
$$

This further gives that

$$
\limsup _{t \rightarrow \infty} \frac{1}{t} \log |X(t)| \leqslant-\frac{c_{1}}{p} \quad \text { a.s.. }
$$


Theorem 3.5. Let Assumption 3.1 hold. Assume that $\mathrm{V}\left(\mathrm{t}_{1}, \mathrm{t}_{2}, \mathrm{x}\right) \in \mathrm{C}^{1,1,2}\left(\mathbb{R}_{+} \times \mathbb{R}_{+} \times \mathbb{R} ; \mathbb{R}_{+}\right)$and $\varphi(\mathrm{t})$ be a nonnegative predictable process, and let $\mathrm{p}>0, \mathrm{c}_{1} \geqslant 0, \mathrm{c}_{2} \geqslant 0, \mathrm{c}_{3}>0, \mathrm{c}_{2}<\frac{\mathrm{c}_{3}}{2}$, and $\rho \geqslant 0, \gamma>0$. Assume that for all $\mathrm{x}_{0} \neq 0$ and $\mathrm{t} \geqslant 0$,

(1) $|x|^{p} \leqslant V\left(t_{1}, t_{2}, x\right)$;

(2) $\mathrm{L}_{1} \mathrm{~V}\left(\mathrm{t}_{1}, \mathrm{t}_{2}, \mathrm{x}\right) \leqslant-\mathrm{c}_{1} \varphi(\mathrm{t}) \mathrm{V}\left(\mathrm{t}_{1}, \mathrm{t}_{2}, \mathrm{x}\right)$;

(3) $\mathrm{L}_{2} \mathrm{~V}\left(\mathrm{t}_{1}, \mathrm{t}_{2}, x\right) \leqslant \mathrm{c}_{2} \varphi(t) \mathrm{V}\left(\mathrm{t}_{1}, \mathrm{t}_{2}, x\right)$;

(4) $\left|V_{x}\left(t_{1}, t_{2}, x\right) \sigma\left(t_{1}, t_{2}, x\right)\right|^{2} \geqslant c_{3} \varphi(t)\left|V\left(t_{1}, t_{2}, x\right)\right|^{2}$;

(5) $\liminf _{\mathrm{t} \rightarrow \infty} \frac{1}{\mathrm{t}} \int_{0}^{\mathrm{t}} \varphi(\mathrm{s}) d \mathrm{E}(\mathrm{s}) \geqslant \rho$ a.s.;

(6) $\liminf _{\mathrm{t} \rightarrow \infty} \frac{1}{\mathrm{t}} \int_{0}^{\mathrm{t}} \varphi(\mathrm{s}) d \mathrm{~s} \geqslant \gamma$ a.s..

Then the solution of equation (2.1) satisfies

$$
\limsup _{t \rightarrow \infty} \frac{1}{t} \log |X(t)| \leqslant-\frac{c_{1}}{p} \gamma-\frac{1}{p}\left(\frac{c_{3}}{2}-c_{2}\right) \rho \text { a.s.. }
$$

Proof. Fix $x_{0} \neq 0$ arbitrarily. It is easy to deduce by time-changed Itô's formula and conditions (2) and (3) that

$$
\begin{aligned}
\log V(t, E(t), X(t)) \leqslant & \log V\left(0,0, x_{0}\right)+c_{1} \int_{0}^{t} \varphi(s) d s+c_{2} \int_{0}^{t} \varphi(s) d E(s) \\
& -\frac{1}{2} \int_{0}^{t} \frac{\left|V_{x}(t, E(t), X(t)) \sigma(t, E(t), X(t))\right|^{2}}{V^{2}(t, E(t), X(t))} d E(s)+M(t),
\end{aligned}
$$

where $M(t)$ has been defined in Theorem 3.4. Let $k=1,2, \ldots$ and assign $0<\varepsilon<1-2 \frac{c_{2}}{c_{3}}$ arbitrarily. In view of Lemma 6.2 in [15] with $g(t)=t, \tau_{k}=k, \gamma_{k}=\varepsilon$, and $\theta=2$, we derive that, for almost all $\omega \in \Omega$, there exists an integer $k_{0}(\omega)$ such that, for all $k>k_{0}, 0 \leqslant t \leqslant k$,

$$
M(t) \leqslant 2 \varepsilon^{-1} \log k+\frac{\varepsilon}{2}\langle M(t)\rangle \leqslant 2 \varepsilon^{-1} \log k+\frac{\varepsilon}{2} \int_{0}^{t} \frac{\left|V_{x}(t, E(t), X(t)) \sigma(t, E(t), X(t))\right|^{2}}{V^{2}(t, E(t), X(t))} d E(s) .
$$

Substituting this into (3.6) and using condition (4) one obtains, for any $0 \leqslant t \leqslant k, k \geqslant k_{0}$,

$$
\begin{aligned}
\log V(t, E(t), X(t)) \leqslant & \log V\left(0,0, x_{0}\right)-c_{1} \int_{0}^{t} \varphi(s) d s+c_{2} \int_{0}^{t} \varphi(s) d E(s)+2 \varepsilon^{-1} \log k \\
& -\frac{1-\varepsilon}{2} \int_{0}^{t} \frac{\left|V_{x}(t, E(t), X(t)) \sigma(t, E(t), X(t))\right|^{2}}{V^{2}(t, E(t), X(t))} d E(s) \\
\leqslant & \log V\left(0,0, x_{0}\right)-c_{1} \int_{0}^{t} \varphi(s) d s+c_{2} \int_{0}^{t} \varphi(s) d E(s)+2 \varepsilon^{-1} \log k-\frac{1-\varepsilon}{2} c_{3} \int_{0}^{t} \varphi(s) d E(s) \\
\leqslant & \log V\left(0,0, x_{0}\right)-c_{1} \int_{0}^{t} \varphi(s) d s+2 \varepsilon^{-1} \log k-\left(\frac{1-\varepsilon}{2} c_{3}-c_{2}\right) \int_{0}^{t} \varphi(s) d E(s)
\end{aligned}
$$

This along with condition (1) implies, for any $0 \leqslant t \leqslant k, k \geqslant k_{0}$,

$$
\begin{aligned}
\frac{1}{t} \log |X(t)| & \leqslant \frac{1}{p t} \log V(t, E(t), X(t)) \\
& \leqslant \frac{1}{p t}\left(\log V\left(0,0, x_{0}\right)-c_{1} \int_{0}^{t} \varphi(s) d s+2 \varepsilon^{-1} \log k-\left(\frac{1-\varepsilon}{2} c_{3}-c_{2}\right) \int_{0}^{t} \varphi(s) d E(s)\right) \\
& \leqslant \frac{1}{p t}\left(\log V\left(0,0, x_{0}\right)-c_{1} \int_{0}^{t} \varphi(s) d s-\left(\frac{1-\varepsilon}{2} c_{3}-c_{2}\right) \int_{0}^{t} \varphi(s) d E(s)\right)+\frac{2 \log k}{\varepsilon p(k-1)} .
\end{aligned}
$$


Consequently

$$
\begin{aligned}
\limsup _{t \rightarrow \infty} \frac{1}{t} \log |X(t)| & \leqslant-\frac{c_{1}}{p} \liminf _{t \rightarrow \infty} \frac{1}{t} \int_{0}^{t} \varphi(s) d s-\frac{1}{p}\left(\frac{1-\varepsilon}{2} c_{3}-c_{2}\right) \liminf _{t \rightarrow \infty} \frac{1}{t} \int_{0}^{t} \varphi(s) d E(s) \\
& \leqslant-\frac{c_{1}}{p} \gamma-\frac{1}{p}\left(\frac{1-\varepsilon}{2} c_{3}-c_{2}\right) \rho \text { a.s.. }
\end{aligned}
$$

Here we have used conditions (5) and (6). Let $\varepsilon \rightarrow 0$. Then we obtain the required result.

In what follows we shall remove hypothesis of Assumption 3.1. However it should be pointed out that the following results are not the generalizations of the previous ones, but cover different case.

Theorem 3.6. Let $\mathrm{V}\left(\mathrm{t}_{1}, \mathrm{t}_{2}, \mathrm{x}\right) \in \mathrm{C}^{1,1,2}\left(\mathbb{R}_{+} \times \mathbb{R}_{+} \times \mathbb{R} ; \mathbb{R}_{+}\right)$and $\mathrm{u}(\mathrm{t})$ be a polynomial with positive coefficients, and let $\mathrm{p}, \lambda$ be positive constants. Assume that for all $\mathrm{x}_{0} \in \mathbb{R}$ and $\mathrm{t} \geqslant 0$,

(1) $|x|^{p} \leqslant V\left(t_{1}, t_{2}, x\right)$;

(2) $\mathrm{L}_{1} \mathrm{~V}\left(\mathrm{t}_{1}, \mathrm{t}_{2}, x\right) \leqslant-\lambda V\left(\mathrm{t}_{1}, \mathrm{t}_{2}, x\right)+\mathrm{u}(\mathrm{t}) e^{-\lambda(t+E(t))}$;

(3) $\mathrm{L}_{2} \mathrm{~V}\left(\mathrm{t}_{1}, \mathrm{t}_{2}, x\right) \leqslant-\lambda \mathrm{V}\left(\mathrm{t}_{1}, \mathrm{t}_{2}, x\right)+\mathrm{u}(\mathrm{t}) e^{-\lambda(\mathrm{t}+\mathrm{E}(\mathrm{t}))}$;

(4) $\left|V_{x}\left(t_{1}, t_{2}, x\right) \sigma\left(t_{1}, t_{2}, x\right)\right|^{2} \leqslant u(t) e^{-\lambda(t+E(t))} V\left(t_{1}, t_{2}, x\right)$;

(5) $\mathrm{E}(\mathrm{t})$ is asymptotically slower than $\mathrm{t}$, i.e., $\lim _{\mathrm{t} \rightarrow \infty} \frac{\mathrm{E}(\mathrm{t})}{\mathrm{t}}=0$ a.s..

Then the solution of equation (2.1) satisfies

$$
\limsup _{t \rightarrow \infty} \frac{1}{t} \log |X(t)| \leqslant-\frac{\lambda}{p} \text { a.s.. }
$$

Proof. Since $\mathrm{E}(\mathrm{t})$ is asymptotically slower than $t$, for any $\varepsilon>0$ we can deduce that there exists a constant $\mathrm{T}>0$ such that

$$
E(t) \leqslant \varepsilon t \quad \text { a.s., }
$$

whenever $t>T$. By time-changed Itô's formula and conditions (2) and (3), we arrive at

$$
\begin{aligned}
e^{\lambda(t+E(t))} V(t, E(t), X(t))= & V\left(0,0, x_{0}\right)+\int_{0}^{t} e^{\lambda(s+E(s))}\left[\lambda V(s, E(s), X(s))+L_{1} V(s, E(s), X(s))\right] d s \\
& +\int_{0}^{t} e^{\lambda(s+E(s))}\left[\lambda V(s, E(s), X(s))+L_{2} V(s, E(s), X(s))\right] d E(s) \\
& +\int_{0}^{t} e^{\lambda(s+E(s))} V_{x}(s, E(s), X(s)) \sigma(s, E(s), X(s)) d B(E(s)) \\
\leqslant & V\left(0,0, x_{0}\right)+\int_{0}^{t} u(s) d s+\int_{0}^{t} u(s) d E(s)+N(t),
\end{aligned}
$$

where

$$
N(t)=\int_{0}^{t} e^{\lambda(s+E(s))} V_{x}(s, E(s), X(s)) \sigma(s, E(s), X(s)) d B(E(s)) .
$$

Let $\frac{q}{2}(q>2)$ be the degree of the polynomial $u(\cdot)$ and $k=1,2, \ldots$ Assign $\theta>\max \left\{1, T^{\frac{1}{k-1}}\right\}$. An application of Lemma 6.2 in [15] with $g(t)=t, \gamma_{k}=\theta^{-q k}, \tau_{k}=\theta^{k}$ yields that, for almost all $\omega \in \Omega$, there exists an integer $k_{0}(\omega)$ such that, for all $k \geqslant k_{0}$ and $0 \leqslant t \leqslant \theta^{k}$,

$$
\begin{aligned}
N(t) & \leqslant \frac{1}{2} \theta^{-q k}\langle N(t)\rangle+\theta^{q k+1} \log k \\
& =\frac{1}{2} \theta^{-q k} \int_{0}^{t} e^{2 \lambda(s+E(s))} V_{x}^{2}(s, E(s), X(s)) \sigma^{2}(s, E(s), X(s)) d E(s)+\theta^{q k+1} \log k .
\end{aligned}
$$


Substituting this into (3.7) and using condition (4) one obtains

$$
\begin{aligned}
e^{\lambda(t+E(t))} V(t, E(t), X(t)) \leqslant & V\left(0,0, x_{0}\right)+\int_{0}^{t} u(s) d s+\int_{0}^{t} u(s) d E(s)+\theta^{q k+1} \log k \\
& +\frac{1}{2} \theta^{-q k} \int_{0}^{t} e^{\lambda(s+E(s))} u(s) V(s, E(s), X(s)) d E(s) \\
\leqslant & V\left(0,0, x_{0}\right)+(u(t))^{2}+\int_{0}^{t} u(s) d E(s)+\theta^{q k+1} \log k+\frac{1}{2} \theta^{-q k} \\
& \times \int_{0}^{t} e^{\lambda(s+E(s))} u(s) V(s, E(s), X(s)) d E(s) \\
= & V\left(0,0, x_{0}\right)+\left(u\left(\theta^{k}\right)\right)^{2}+\theta^{q k+1} \log k \\
& +\int_{0}^{t} u(s)\left[1+\frac{1}{2} \theta^{-q k} \times e^{\lambda(s+E(s))} V(s, E(s), X(s))\right] d E(s),
\end{aligned}
$$

which further gives that

$$
\begin{aligned}
1+\frac{1}{2} \theta^{-q k} e^{\lambda(t+E(t))} V(t, E(t), X(t)) \leqslant & +\frac{1}{2} \theta^{-q k} V\left(0,0, x_{0}\right)+\frac{1}{2} \theta^{-q k}\left(u\left(\theta^{k}\right)\right)^{2}+\frac{1}{2} \theta \log k \\
& +\frac{1}{2} \theta^{-q k} \int_{0}^{t} u(s)\left[1+\frac{1}{2} \theta^{-q k} e^{\lambda(s+E(s))} V(s, E(s), X(s))\right] d E(s) .
\end{aligned}
$$

By time-changed Gronwall's inequality in [20], we deduce

$$
\begin{aligned}
1+\frac{1}{2} \theta^{-q k} e^{\lambda(t+E(t))} V(t, E(t), X(t)) \leqslant & \left(1+\frac{1}{2} \theta^{-q k} V\left(0,0, x_{0}\right)+\frac{1}{2} \theta^{-q k}\left(u\left(\theta^{k}\right)\right)^{2}+\frac{1}{2} \theta \log k\right) \\
& \times \exp \left\{\frac{1}{2} \theta^{-q k} \int_{0}^{t} u(s) d E(s)\right\} .
\end{aligned}
$$

This, together with condition (5) and the monotonicity of $u$ and $E$, implies that, for all $T \leqslant t \leqslant \theta^{k}, k \geqslant k_{0}$,

$$
\begin{aligned}
e^{\lambda(t+E(t))} V(t, E(t), X(t)) & \leqslant\left(2 \theta^{q k}+V\left(0,0, x_{0}\right)+\left(u\left(\theta^{k}\right)\right)^{2}+\theta^{q k+1} \log k\right) \times \exp \left\{\frac{1}{2} \theta^{-q k} \int_{0}^{t} u(s) d E(s)\right\} \\
& \leqslant\left(2 \theta^{q k}+V\left(0,0, x_{0}\right)+\left(u\left(\theta^{k}\right)\right)^{2}+\theta^{q k+1} \log k\right) \times \exp \left\{\frac{1}{2} \theta^{-(q-1) k} \varepsilon u\left(\theta^{k}\right)\right\} \\
& \leqslant C \theta^{q k+1} \log k \text { a.s., }
\end{aligned}
$$

where $C$ is a finite random variable independent of $k$. If $\theta^{k-1}<t<\theta^{k}, k \geqslant k_{0}$,

$$
\frac{\mathrm{e}^{\lambda(t+E(t))} V(t, E(t), X(t))}{t^{q} \log \log t} \leqslant C \theta^{q k+1} \frac{\log k}{\theta^{q(k-1)} \log \log \theta^{k-1}} \leqslant C \theta^{q+1} \frac{\log k}{\log (k-1)+\log \log \theta},
$$

which implies

$$
\limsup _{t \rightarrow \infty} \frac{e^{\lambda(t+E(t))} V(t, E(t), X(t))}{t^{q} \log \log t} \leqslant C \theta^{q+1} \text { a.s.. }
$$

Since $\theta>1$ is arbitrary,

$$
\limsup _{t \rightarrow \infty} \frac{e^{\lambda(t+E(t))} V(t, E(t), X(t))}{t^{q} \log \log t} \leqslant C \text { a.s.. }
$$

Finally, by condition (1)

$$
\limsup _{t \rightarrow \infty} \frac{1}{t} \log |X(t)| \leqslant \limsup _{t \rightarrow \infty} \frac{1}{p t} \log V(t, E(t), X(t))
$$




$$
\begin{aligned}
& =\limsup _{t \rightarrow \infty} \frac{1}{p t} \log \left(e^{-\lambda(t+E(t))} \frac{e^{\lambda(t+E(t))} V(t, E(t), X(t))}{t^{q} \log \log t} t^{q} \log \log t\right) \\
& \leqslant-\frac{\lambda}{p} \liminf _{t \rightarrow \infty} \frac{E(t)}{t}-\frac{\lambda}{p} \leqslant-\frac{\lambda}{p} \text { a.s.. }
\end{aligned}
$$

Theorem 3.7. Let $\mathrm{V}\left(\mathrm{t}_{1}, \mathrm{t}_{2}, \mathrm{x}\right) \in \mathrm{C}^{1,1,2}\left(\mathbb{R}_{+} \times \mathbb{R}_{+} \times \mathbb{R} ; \mathbb{R}_{+}\right)$and $\varphi(\mathrm{t})$ be a nonnegative predictable process. Let $\mathrm{p}$, $q, \lambda, \eta$ be positive constants and $\alpha \in[0,1)$. Assume that for all $x_{0} \in \mathbb{R}$ and $\mathrm{t} \geqslant 0$,

(1) $e^{t_{1}+t_{2}}|x|^{p} \leqslant V\left(t_{1}, t_{2}, x\right)$;

(2) $\mathrm{L}_{1} \mathrm{~V}\left(\mathrm{t}_{1}, \mathrm{t}_{2}, x\right) \leqslant 0$;

(3) $L_{2} V\left(t_{1}, t_{2}, x\right)+\eta(1+t)^{-q}\left|V_{x}\left(t_{1}, t_{2}, x\right) \sigma\left(t_{1}, t_{2}, x\right)\right|^{2} \leqslant \varphi(t)\left[1+\left(V\left(t_{1}, t_{2}, x\right)\right)^{\alpha}\right]$;

(4) $\limsup _{\mathrm{t} \rightarrow \infty} \frac{1}{\mathrm{t}} \log \left(\int_{0}^{\mathrm{t}} \varphi(\mathrm{s}) d \mathrm{E}(\mathrm{s})\right) \leqslant 0$ a.s.;

(5) $\mathrm{E}(\mathrm{t})$ is asymptotically slower than $\mathrm{t}$, i.e., $\lim _{\mathrm{t} \rightarrow \infty} \frac{\mathrm{E}(\mathrm{t})}{\mathrm{t}}=0$ a.s..

Then the solution of equation (2.1) satisfies

$$
\limsup _{t \rightarrow \infty} \frac{1}{t} \log |X(t)| \leqslant-\frac{\lambda}{p} \text { a.s.. }
$$

Proof. Fix any $t \geqslant 0$ and $x_{0}$. By time-changed Itô's formula,

$$
\begin{aligned}
V(t, E(t), X(t))= & V\left(0,0, x_{0}\right)+\int_{0}^{t} L_{1} V(s, E(s), X(s)) d s+\int_{0}^{t} L_{2} V(s, E(s), X(s)) d E(s) \\
& +\int_{0}^{t} V_{x}(s, E(s), X(s)) \sigma(s, E(s), X(s)) d B(E(s)) .
\end{aligned}
$$

Let $k=1,2, \ldots$. An application of Lemma 6.2 in [15] with $g(t)=t, \gamma_{k}=2 \eta\left(1+2^{k}\right)^{-q}, \tau_{k}=2^{k}$ and $\theta=2$ yields that, for almost all $\omega \in \Omega$, there exists an integer $k_{0}(\omega)$ such that for all $k \geqslant k_{0}$ and $0 \leqslant t \leqslant 2^{k}$

$$
\bar{N}(t) \leqslant \frac{1}{\eta}\left(1+2^{k}\right)^{q} \log k+\eta\left(1+2^{k}\right)^{-q} \int_{0}^{t} V_{x}^{2}(s, E(s), X(s)) \sigma^{2}(s, E(s), X(s)) d E(s),
$$

where

$$
\overline{\mathrm{N}}(\mathrm{t})=\int_{0}^{\mathrm{t}} \mathrm{V}_{x}(\mathrm{~s}, \mathrm{E}(\mathrm{s}), X(\mathrm{~s})) \sigma(s, \mathrm{E}(\mathrm{s}), X(\mathrm{~s})) \mathrm{dB}(\mathrm{E}(\mathrm{s}))
$$

Substituting this into (3.8) and using conditions (2) and (3), one obtains, for all $0 \leqslant t \leqslant 2^{k}, k \geqslant k_{0}$,

$$
\begin{aligned}
V(t, E(t), X(t)) \leqslant & V\left(0,0, x_{0}\right)+\int_{0}^{t} L_{1} V(s, E(s), X(s)) d s+\int_{0}^{t} L_{2} V(s, E(s), X(s)) d E(s) \\
& +\frac{1}{\eta}\left(1+2^{k}\right)^{q} \log k+\eta\left(1+2^{k}\right)-q \int_{0}^{t} V_{x}^{2}(s, E(s), X(s)) \sigma^{2}(s, E(s), X(s)) d E(s) \\
\leqslant & V\left(0,0, x_{0}\right)+\int_{0}^{t} L_{1} V(s, E(s), X(s)) d s+\frac{1}{\eta}\left(1+2^{k}\right)^{q} \log k \\
& +\int_{0}^{t}\left[L_{2} V(s, E(s), X(s))+\eta\left(1+2^{k}\right)^{-q} V_{x}^{2}(s, E(s), X(s)) \sigma^{2}(s, E(s), X(s))\right] d E(s) \\
\leqslant & V\left(0,0, x_{0}\right)+\int_{0}^{t} L_{1} V(s, E(s), X(s)) d s+\frac{1}{\eta}\left(1+2^{k}\right)^{q} \log k \\
& +\int_{0}^{t}\left[L_{2} V(s, E(s), X(s))+\eta(1+s)^{-q} V_{x}^{2}(s, E(s), X(s)) \sigma^{2}(s, E(s), X(s))\right] d E(s) \\
\leqslant & V\left(0,0, x_{0}\right)+\int_{0}^{t} L_{1} V(s, E(s), X(s)) d s+\frac{1}{\eta}\left(1+2^{k}\right)^{q} \log k
\end{aligned}
$$




$$
\begin{aligned}
& +\int_{0}^{t} \varphi(s)\left[1+(\mathrm{V}(\mathrm{s}, \mathrm{E}(\mathrm{s}), \mathrm{X}(\mathrm{s})))^{\alpha}\right] \mathrm{dE}(\mathrm{s}) \\
\leqslant & \mathrm{V}\left(0,0, \mathrm{x}_{0}\right)+\frac{1}{\eta}\left(1+2^{\mathrm{k}}\right)^{\mathrm{q}} \log \mathrm{k}+\int_{0}^{\mathrm{t}} \varphi(\mathrm{s})\left[1+(\mathrm{V}(\mathrm{s}, \mathrm{E}(\mathrm{s}), \mathrm{X}(\mathrm{s})))^{\alpha}\right] \mathrm{dE}(\mathrm{s}) \text { a.s.. }
\end{aligned}
$$

Now applying Corollary 1.7.5 in [15] we derive

$$
V(t, E(t), X(t)) \leqslant\left[\left(V\left(0,0, x_{0}\right)+\frac{1}{\eta}\left(1+2^{k}\right)^{q} \log k+\int_{0}^{2^{k}} \varphi(s) d E(s)\right)^{1-\alpha}+(1-\alpha) \int_{0}^{2^{k}} \varphi(s) d E(s)\right]^{\frac{1}{1-\alpha}}
$$

for all $0 \leqslant t \leqslant 2^{k}, k \geqslant k_{0}$ almost surely. Now let $\varepsilon>0$ be arbitrary and by condition (4) one sees that there exists a random integer $k_{1}=k_{1}(\omega)$ such that for all $k \geqslant k_{1}$,

$$
\int_{0}^{2^{k}} \varphi(s) \mathrm{dE}(s) \leqslant \mathrm{e}^{\varepsilon^{k}} \text { a.s.. }
$$

Therefore, if $2^{k-1} \leqslant t \leqslant 2^{k}, k \geqslant k_{0} \vee k_{1}$

$$
\begin{aligned}
\frac{1}{t} \log \left(e^{\lambda(t+E(t))}|X(t)|^{p}\right) \leqslant & \frac{1}{t} \log V(t, E(t), X(t)) \\
\leqslant & \frac{1}{t(1-\alpha)} \log \left[\left(V\left(0,0, x_{0}\right)+\frac{1}{\eta}\left(1+2^{k}\right)^{q} \log k+\int_{0}^{2^{k}} \varphi(s) d E(s)\right)^{1-\alpha}\right. \\
& \left.+(1-\alpha) \int_{0}^{2^{k}} \varphi(s) d E(s)\right] \\
\leqslant & \frac{1}{2^{k-1}(1-\alpha)} \log \left[\left(V\left(0,0, x_{0}\right)+\frac{1}{\eta}\left(1+2^{k}\right)^{q} \log k+e^{\varepsilon 2^{k}}\right)^{1-\alpha}+(1-\alpha) e^{\varepsilon 2^{k}}\right],
\end{aligned}
$$

where condition (1) has been used. This immediately implies

$$
\limsup _{t \rightarrow \infty} \frac{1}{t} \log \left(e^{\lambda(t+E(t))}|X(t)|^{p}\right) \leqslant \frac{2 \varepsilon}{1-\alpha} \text { a.s.. }
$$

Since $\varepsilon>0$ is arbitrary,

$$
\limsup _{t \rightarrow \infty} \frac{1}{t} \log \left(e^{\lambda(t+E(t))}|X(t)|^{p}\right) \leqslant 0 \text { a.s.. }
$$

Finally,

$$
\begin{aligned}
\limsup _{t \rightarrow \infty} \frac{1}{t} \log |X(t)| & =\limsup _{t \rightarrow \infty} \frac{1}{p t} \log \left(e^{-\lambda(t+E(t))} e^{\lambda(t+E(t))}|X(t)|^{p}\right) \\
& \leqslant-\frac{\lambda}{p}-\frac{\lambda}{p} \liminf _{t \rightarrow \infty} \frac{E(t)}{t} \leqslant-\frac{\lambda}{p} \text { a.s.. }
\end{aligned}
$$

Theorem 3.8. Let $\mathrm{V}\left(\mathrm{t}_{1}, \mathrm{t}_{2}, \mathrm{x}\right) \in \mathrm{C}^{1,1,2}\left(\mathbb{R}_{+} \times \mathbb{R}_{+} \times \mathbb{R} ; \mathbb{R}_{+}\right)$and $\varphi_{1}(\mathrm{t}), \varphi_{2}(\mathrm{t})$ be two nonnegative predictable processes. Let $\mathrm{p}, \mathrm{q}, \lambda, \eta$ be positive constants and $\alpha \in[0,1)$. Assume that for all $\mathrm{x}_{0} \in \mathbb{R}$ and $\mathrm{t} \geqslant 0$,

(1) $e^{t_{1}+t_{2}}|x|^{p} \leqslant V\left(t_{1}, t_{2}, x\right)$;

(2) $\mathrm{L}_{1} \mathrm{~V}\left(\mathrm{t}_{1}, \mathrm{t}_{2}, x\right) \leqslant 0$;

(3) $\mathrm{L}_{2} \mathrm{~V}\left(\mathrm{t}_{1}, \mathrm{t}_{2}, x\right)+\eta e^{-\mathrm{qt}}\left|\mathrm{V}_{\mathrm{x}}\left(\mathrm{t}_{1}, \mathrm{t}_{2}, x\right) \sigma\left(\mathrm{t}_{1}, \mathrm{t}_{2}, x\right)\right|^{2} \leqslant \varphi_{1}(\mathrm{t})+\varphi_{2}(\mathrm{t})\left(\mathrm{V}\left(\mathrm{t}_{1}, \mathrm{t}_{2}, x\right)\right)^{\alpha}$;

(4) $\limsup _{\mathrm{t} \rightarrow \infty} \frac{1}{\mathrm{t}} \log \left(\int_{0}^{\mathrm{t}} \varphi_{1}(\mathrm{~s}) d \mathrm{E}(\mathrm{s})\right) \leqslant \mathrm{q}$ a.s.;

(5) $\lim \sup _{\mathrm{t} \rightarrow \infty} \frac{1}{\mathrm{t}} \log \left(\int_{0}^{\mathrm{t}} \varphi_{2}(\mathrm{~s}) d \mathrm{E}(\mathrm{s})\right) \leqslant(1-\alpha) \mathrm{q}$ a.s.; 
(6) $\mathrm{E}(\mathrm{t})$ is asymptotically slower than $\mathrm{t}$, i.e., $\lim _{\mathrm{t} \rightarrow \infty} \frac{\mathrm{E}(\mathrm{t})}{\mathrm{t}}=0$ a.s..

Then the solution of equation (2.1) satisfies

$$
\limsup _{t \rightarrow \infty} \frac{1}{t} \log |X(t)| \leqslant-\frac{1}{p}(\lambda-q) \text { a.s.. }
$$

Proof. According to (3.8) we have

$$
V(t, E(t), X(t))=V\left(0,0, x_{0}\right)+\int_{0}^{t} L_{1} V(s, E(s), X(s)) d s+\int_{0}^{t} L_{2} V(s, E(s), X(s)) d E(s)+\bar{N}(t)
$$

Let $k=1,2, \ldots$. Again making use of Lemma 6.2 in [15] with $g(t)=t, \gamma_{k}=2 \eta e^{-q k}, \tau_{k}=k$, and $\theta=2$ one can derive that, for almost all $\omega \in \Omega$, there exists an integer $k_{0}(\omega)$ such that for all $k \geqslant k_{0}$ and $0 \leqslant t \leqslant k$

$$
\bar{N}(t) \leqslant \frac{1}{\eta} e^{q k} \log k+\eta e^{-q k} \int_{0}^{t} V_{x}^{2}(s, E(s), X(s)) \sigma^{2}(s, E(s), X(s)) d E(s) .
$$

Substituting this into (3.9) and using conditions (2) and (3), one obtains

$$
\begin{aligned}
V(t, E(t), X(t)) \leqslant & V\left(0,0, x_{0}\right)+\int_{0}^{t} L_{1} V(s, E(s), X(s)) d s+\int_{0}^{t} L_{2} V(s, E(s), X(s)) d E(s) \\
& +\frac{1}{\eta} e^{q k} \log k+\eta e^{-q k} \int_{0}^{t} V_{x}^{2}(s, E(s), X(s)) \sigma^{2}(s, E(s), X(s)) d E(s) \\
\leqslant & V\left(0,0, x_{0}\right)+\frac{1}{\eta} e^{q k} \log k+\int_{0}^{t}\left[\varphi_{1}(s)+\varphi_{2}(s)(V(s, E(s), X(s)))^{\alpha}\right] d E(s)
\end{aligned}
$$

for all $0 \leqslant t \leqslant k, k \geqslant k_{0}$ almost surely. So by Corollary 1.7 .5 in [15] we deduce

$$
\begin{aligned}
V(t, E(t), X(t)) & \leqslant V\left(0,0, x_{0}\right)+\frac{1}{\eta} e^{q k} \log k+\int_{0}^{k} \varphi_{1}(s) d E(s)+\int_{0}^{t} \varphi_{2}(s)(V(s, E(s), X(s)))^{\alpha} d E(s) \\
& \leqslant\left[\left(V\left(0,0, x_{0}\right)+\frac{1}{\eta} e^{q k} \log k+\int_{0}^{k} \varphi_{1}(s) d E(s)\right)^{1-\alpha}+(1-\alpha) \int_{0}^{k} \varphi_{2}(s) d E(s)\right]^{\frac{1}{1-\alpha}}
\end{aligned}
$$

for all $0 \leqslant t \leqslant k, k \geqslant k_{0}$ almost surely. Let $\varepsilon>0$ be arbitrary. By conditions (4) and (5) there exists a random integer $k_{1}=k_{1}(\omega)$ such that

$$
\int_{0}^{k} \varphi_{1}(s) \mathrm{dE}(\mathrm{s}) \leqslant \mathrm{e}^{(\mathrm{q}+\varepsilon) \mathrm{k}} \text { and } \int_{0}^{\mathrm{k}} \varphi_{2}(\mathrm{~s}) \mathrm{dE}(\mathrm{s}) \leqslant \mathrm{e}^{(1-\alpha)(\mathrm{q}+\varepsilon) \mathrm{k}}
$$

for all $k \geqslant k_{1}$. Hence, if $k-1 \leqslant t \leqslant k, k \geqslant k_{0} \vee k_{1}$,

$$
V(t, E(t), X(t)) \leqslant\left[\left(V\left(0,0, x_{0}\right)+\frac{1}{\eta} e^{q k} \log k+e^{(q+\varepsilon) k}\right)^{1-\alpha}+(1-\alpha) e^{(1-\alpha)(q+\varepsilon) k}\right]^{\frac{1}{1-\alpha}},
$$

which implies immediately that

$$
\limsup _{t \rightarrow \infty} \frac{1}{t} \log V(t, E(t), X(t)) \leqslant q+\varepsilon \text { a.s.. }
$$

Letting $\varepsilon \rightarrow 0$, it gives

$$
\limsup _{t \rightarrow \infty} \frac{1}{t} \log V(t, E(t), X(t)) \leqslant q \text { a.s.. }
$$


Finally,

$$
\begin{aligned}
\limsup _{t \rightarrow \infty} \frac{1}{t} \log |X(t)| & \leqslant \limsup _{t \rightarrow \infty} \frac{1}{p t} \log \left[e^{-\lambda(t+E(t))} V(t, E(t), X(t))\right] \\
& \leqslant \frac{q}{p}-\frac{\lambda}{p}-\frac{\lambda}{p} \liminf _{t \rightarrow \infty} \frac{E(t)}{t} \leqslant-\frac{\lambda}{p}+\frac{q}{p}=-\frac{1}{p}(\lambda-q) \text { a.s. }
\end{aligned}
$$

as required. The proof is complete.

The following theorem gives a sufficient condition for a stochastic system which is almost surely exponentially instable.

Theorem 3.9. Let Assumption 3.1 hold. Let $\mathrm{V}\left(\mathrm{t}_{1}, \mathrm{t}_{2}, \mathrm{x}\right) \in \mathrm{C}^{1,1,2}\left(\mathbb{R}_{+} \times \mathbb{R}_{+} \times \mathbb{R}_{;} \mathbb{R}_{+}\right)$and $\varphi(\mathrm{t})$ be a nonnegative predictable process, and $\mathrm{p}>0, \mathrm{c}_{1} \geqslant 0, \mathrm{c}_{2}>0, \mathrm{c}_{3}>0, \mathrm{c}_{2}>\frac{\mathrm{c}_{3}}{2}$, and $\rho \geqslant 0, \gamma>0$. Assume that for all $\mathrm{x}_{0} \neq 0$ and $t \geqslant 0$,

(1) $|x|^{p} \geqslant V\left(t_{1}, t_{2}, x\right)$;

(2) $\mathrm{L}_{1} \mathrm{~V}\left(\mathrm{t}_{1}, \mathrm{t}_{2}, x\right) \geqslant \mathrm{c}_{1} \varphi(t) \mathrm{V}\left(\mathrm{t}_{1}, \mathrm{t}_{2}, x\right)$;

(3) $L_{2} V\left(t_{1}, t_{2}, x\right) \geqslant c_{2} \varphi(t) V\left(t_{1}, t_{2}, x\right)$;

(4) $\left|V_{x}\left(t_{1}, t_{2}, x\right) \sigma\left(t_{1}, t_{2}, x\right)\right|^{2} \geqslant c_{3} \varphi(t)\left|V\left(t_{1}, t_{2}, x\right)\right|^{2}$;

(5) $\liminf _{\mathrm{t} \rightarrow \infty} \frac{1}{\mathrm{t}} \int_{0}^{\mathrm{t}} \varphi(\mathrm{s}) d \mathrm{E}(\mathrm{s}) \geqslant \rho$ a.s.;

(6) $\liminf _{\mathrm{t} \rightarrow \infty} \frac{1}{\mathrm{t}} \int_{0}^{\mathrm{t}} \varphi(\mathrm{s}) d \mathrm{~s} \geqslant \gamma$ a.s..

Then the solution of equation (2.1) satisfies

$$
\liminf _{t \rightarrow \infty} \frac{1}{t} \log |X(t)| \geqslant \frac{c_{1} \gamma}{p}+\frac{1}{p}\left(c_{2}-\frac{c_{3}}{2}\right) \rho \text { a.s.. }
$$

Proof. Fix $x_{0} \neq 0$ arbitrarily. It is easy to deduce by time-changed Itô's formula and conditions (2), (3), and (4) that

$$
\log V(t, E(t), X(t)) \geqslant \log V\left(0,0, x_{0}\right)+c_{1} \int_{0}^{t} \varphi(s) d s+c_{2} \int_{0}^{t} \varphi(s) d E(s)-\frac{c_{3}}{2} \int_{0}^{t} \varphi(s) d E(s)+M(t),
$$

where $M(t)$ has been defined in Theorem 3.4. Let $k=1,2, \ldots$ and assign $0<\varepsilon<2 \frac{c_{2}}{c_{3}}-1$ arbitrarily. Applying Lemma 6.2 in [15] to the martingale $-M(t)$ with $g(t)=t, \tau_{k}=k, \gamma_{k}=\varepsilon$, and $\theta=2$, we deduce that, for almost all $\omega \in \Omega$, there exists an integer $k_{0}(\omega)$ such that for all $k>k_{0}$

$$
-M(t) \leqslant 2 \varepsilon^{-1} \log k+\frac{\varepsilon}{2}\langle M(t)\rangle \leqslant 2 \varepsilon^{-1} \log k+\frac{\varepsilon}{2} \int_{0}^{t} \frac{\left|V_{x}(t, E(t), X(t)) \sigma(t, E(t), X(t))\right|^{2}}{V^{2}(t, E(t), X(t))} d E(s)
$$

for all $0 \leqslant t \leqslant k$. Substituting this into (3.10) and using condition (4) one obtains

$$
\begin{aligned}
\log \mathrm{V}(\mathrm{t}, \mathrm{E}(\mathrm{t}), \mathrm{X}(\mathrm{t})) \geqslant & \log \mathrm{V}\left(0,0, \mathrm{x}_{0}\right)+\mathrm{c}_{1} \int_{0}^{\mathrm{t}} \varphi(\mathrm{s}) \mathrm{d} s+\left(\mathrm{c}_{2}-\frac{\mathrm{c}_{3}}{2}\right) \int_{0}^{\mathrm{t}} \varphi(s) \mathrm{dE}(\mathrm{s})-2 \varepsilon^{-1} \log \mathrm{k} \\
& -\frac{\varepsilon}{2} \int_{0}^{\mathrm{t}} \frac{\left|\mathrm{V}_{\mathrm{x}}(\mathrm{t}, \mathrm{E}(\mathrm{t}), \mathrm{X}(\mathrm{t})) \sigma(\mathrm{t}, \mathrm{E}(\mathrm{t}), \mathrm{X}(\mathrm{t}))\right|^{2}}{\mathrm{~V}^{2}(\mathrm{t}, \mathrm{E}(\mathrm{t}), \mathrm{X}(\mathrm{t}))} \mathrm{dE}(\mathrm{s}) \\
\geqslant & \log \mathrm{V}\left(0,0, \mathrm{x}_{0}\right)+\mathrm{c}_{1} \int_{0}^{\mathrm{t}} \varphi(\mathrm{s}) \mathrm{d} \mathrm{s}+\left(\mathrm{c}_{2}-\frac{(1+\varepsilon) \mathrm{c}_{3}}{2}\right) \int_{0}^{\mathrm{t}} \varphi(\mathrm{s}) \mathrm{dE}(\mathrm{s})-2 \varepsilon^{-1} \log \mathrm{k}
\end{aligned}
$$

for all $0 \leqslant t \leqslant k, k \geqslant k_{0}$ almost surely. This, together with conditions (1), (5), and (6), implies

$$
\liminf _{t \rightarrow \infty} \frac{1}{t} \log |X(t)| \geqslant \frac{c_{1} \gamma}{p}+\frac{1}{p}\left(c_{2}-\frac{1+\varepsilon}{2} c_{3}\right) \rho \text { a.s.. }
$$

Since $\varepsilon$ is arbitrary, the conclusion follows and the proof is complete. 


\section{An illustrative example}

In this section, we consider an example to verify the stability of time-changed SDEs. We assume that all coefficients of SDEs satisfy Assumptions 2.1, 2.2, and 3.1.

Example 4.1. Consider a one-dimensional inhomogeneous linear SDE driven by time-changed Brownian motion

$$
d X(t)=-\alpha X(t) d t+\beta X(t) d E(t)+\sigma X(t) d B(E(t)), X_{0}=x_{0} \neq 0,
$$

where $\alpha, \beta, \sigma>0$. In the following section, we will analyse the almost sure exponential stability of (4.1). Taking Lyapunov function $V\left(t_{1}, t_{2}, x\right)=|x|^{2}$, we have

$$
\begin{aligned}
& \mathrm{L}_{1} \mathrm{~V}(\mathrm{t}, \mathrm{E}(\mathrm{t}), \mathrm{X}(\mathrm{t}))=-2 \alpha|\mathrm{X}(\mathrm{t})|^{2}=-2 \alpha \mathrm{V}(\mathrm{t}, \mathrm{E}(\mathrm{t}), \mathrm{X}(\mathrm{t})), \\
& \mathrm{L}_{2} \mathrm{~V}(\mathrm{t}, \mathrm{E}(\mathrm{t}), \mathrm{X}(\mathrm{t}))=\left(2 \beta+\sigma^{2}\right)|\mathrm{X}(\mathrm{t})|^{2}=\left(2 \beta+\sigma^{2}\right) \mathrm{V}(\mathrm{t}, \mathrm{E}(\mathrm{t}), \mathrm{X}(\mathrm{t}))
\end{aligned}
$$

and

$$
\left|V_{x}(t, E(t), X(t)) \sigma(t, E(t), X(t))\right|^{2}=4 \sigma^{2}|X(t)|^{2}=4 \sigma^{2} \mid V\left(t, E(t),\left.X(t)\right|^{2}\right.
$$

Let $p=2, c_{1}=2 \alpha, c_{2}=2 \beta+\sigma^{2}, c_{3}=4 \sigma^{2}, \gamma=1$. Therefore, if $\sigma^{2}>2 \beta$, applying Theorem 3.5 , we obtain

$$
\limsup _{t \rightarrow \infty} \frac{1}{t} \log |X(t)| \leqslant-\alpha-\frac{1}{2}\left(\sigma^{2}-2 \beta\right) \rho \text { a.s., }
$$

that is to say, the solution of (4.1) is almost surely exponentially stable.

\section{Acknowledgment}

We are grateful to the referees for their many valuable comments and suggestions which improved this article. This work was supported by the National Natural Sciences Foundations of China (No. 11371374, No. 11771452, No. 11571372), the Natural Science Foundation of Hunan University of Technology (2012HZX08), and the Natural Sciences Foundations of Hunan (No. 2017JJ2328).

\section{References}

[1] J.-H. Bao, Z.-T. Hou, C.-G. Yuan, Stability in distribution of mild solutions to stochastic partial differential equations, Proc. Amer. Math. Soc., 138 (2010), 2169-2180. 1

[2] J.-H. Bao, A. Truman, C.-G. Yuan, Stability in distribution of mild solutions to stochastic partial differential delay equations with jumps, Proc. R. Soc. Lond. Ser. A Math. Phys. Eng. Sci., 465 (2009), 2111-2134.

[3] J.-H. Bao, A. Truman, C.-G. Yuan, Almost sure asymptotic stability of stochastic partial differential equations with jumps, SIAM J. Control Optim., 49 (2011), 771-787. 1

[4] P. Carra, L.-R. Wu, Time-changed Lévy processes and option pricing, J. Financial Econ., 71 (2004), 113-141. 1

[5] H. Geman, D.-B. Madan, M. Yor, Time changes for Lévy processes, Math. Finance, 11 (2001), 79-96.

[6] M.-G. Hahn, K. Kobayashi, J. Ryvkina, S. Umarov, On time-changed Gaussian processes and their associated FokkerPlanck-Kolmogorov equations, Electron. Commun. Probab., 16 (2011), 150-164. 1

[7] M. Hahn, K. Kobayashi, S. Umarov, SDEs driven by a time-changed Lévy process and their associated time-fractional order pseudo-differential equations, J. Theoret. Probab., 25 (2012), 262-279. 1

[8] Z.-T. Hou, J.-H. Bao, C.-G. Yuan, Exponential stability of energy solutions to stochastic partial differential equations with variable delays and jumps, J. Math. Anal. Appl., 366 (2010), 44-54. 1

[9] E. Jum, K. Kobayashi, A strong and weak approximation scheme for stochastic differential equations driven by a timechanged Brownian motion, Probab. Math. Statist., 2014 (2014), 201-220. 1, 3

[10] J. Kallsen, A. N. Shiryaev, Time change representation of stochastic integrals, translated from Teor. Veroyatnost. i Primenen., 46 (2001), 579-585, Theory Probab. Appl., 46 (2003), 522-528. 1

[11] K. Kobayashi, Stochastic calculus for a time-changed semimartingale and the associated stochastic differential equations, J. Theoret. Probab., 24 (2011), 789-820. 1, 2

[12] H.-H. Kuo, Introduction to stochastic integration, Universitext, Springer, New York, (2006). 3

[13] M. Magdziarz, Path properties of subdiffusion-a martingale approach, Stoch. Models, 26 (2010), 256-271. 1, 3

[14] M. Magdziarz, R. L. Schilling, Asymptotic properties of Brownian motion delayed by inverse subordinators, Proc. Amer. Math. Soc., 143 (2015), 4485-4501. 1, 2 
[15] X.-R. Mao, Exponential stability of stochastic differential equations, Monographs and Textbooks in Pure and Applied Mathematics, Marcel Dekker, Inc., New York, (1994). 1, 3, 3, 3, 3, 3, 3

[16] X.-R. Mao, Stochastic differential equations and applications, Second edition, Horwood Publishing Limited, Chichester, (2008). 1, 3

[17] E. Nane, Y.-N. Ni, Stability of the solution of stochastic differential equation driven by time-changed Léry noise, Proc. Amer. Math. Soc., 145 (2017), 3085-3104. 1

[18] P. E. Protter, Stochastic integration and differential equations, Second edition, Applications of Mathematics (New York), Stochastic Modelling and Applied Probability, Springer-Verlag, Berlin, (2004). 2

[19] L. Tan, W. Jin, Y.-Q. Suo, Stability in distribution of neutral stochastic functional differential equations, Statist. Probab. Lett., 107 (2015), 27-36. 1

[20] Q. Wu, Stability analysis for a class of nonlinear time-changed systems, Cogent Math., 3 (2016), 10 pages. 1, 3

[21] Q. Wu, Stability of stochastic differential equations with respect to time-changed Brownian motions, ArXiv, 2016 (2016), 22 pages. 1,3

[22] W.-N. Zhou, J. Yang, X.-Q. Yang, A. Dai, H.-S. Liu, J.-A. Fang, pth moment exponential stability of stochastic delayed hybrid systems with Lévy noise, Appl. Math. Model., 39 (2015), 5650-5658. 1

[23] Q.-X. Zhu, Asymptotic stability in the pth moment for stochastic differential equations with Léry noise, J. Math. Anal. Appl., 416 (2014), 126-142. 1 